\title{
How to Train to Discharge a Dermatology Outpatient: A Review
}

\author{
A. Harun ${ }^{a}$ Andrew Y. Finlay ${ }^{a}$ M. Sam Salek ${ }^{b, c}$ Vincent Piguet ${ }^{a, d, e}$ \\ a Dermatology and Academic Wound Healing, Division of Infection and Immunity, School of Medicine, \\ Cardiff University, and ${ }^{b}$ Institute for Medicines Development, Cardiff, ' School of Life and Medical Sciences, \\ Department of Pharmacy, University of Hertfordshire, Hatfield, UK; ${ }^{d}$ Division of Dermatology, Women's College \\ Hospital and ${ }^{e}$ Division of Dermatology, Department of Medicine, University of Toronto, Toronto, ON, Canada
}

\section{Keywords}

Education - Discharge decision - Dermatology outpatients . Clinical practice

\begin{abstract}
Background/Aims: The decision to discharge is a critical and common outpatient consultation event. However, little guidance exists over how discharge decision-making can be taught. We aimed to provide educational recommendations concerning outpatient discharge decision-making. Methods: Recommendations were drawn from prior interviews with 40 consultant dermatologists and 56 dermatology outpatients, and from the "traffic light" design discharge information checklist, developed using the Delphi technique. Results: The key strategies to follow to appropriately manage the outpatient discharge process are: to warn patients in advance, to understand patients' agendas, to allow extra time for the discharge process, to prepare patients to self-manage, to provide a "safety net" and provide the GP with a clear management plan. Aspects to be considered include patient mobility, presence of carer, type of employment, diagnostic certainty, and use of the checklist or guidelines. Key training aspects include teaching structured thought processes when discharging, discharging according to context, developing communication and negotiation
\end{abstract}

\section{KARGER}

(C) 2017 S. Karger AG, Basel

E-Mail karger@karger.com

www.karger.com/drm skills, avoiding decision biases and encouraging good interprofessional collaboration. Training should include the consideration of the possibility of discharge at each consultation. Novel training strategies have been developed on how to appropriately manage the outpatient discharge process, including involving and informing patients. These strategies focus on safe decision-making, being patient-centred and organizing an efficient health care service framework. Conclusion: Structured outpatient discharge training for dermatologists is now possible, based on information from detailed doctor- and patient-based qualitative studies.

(c) 2017 S. Karger AG, Basel

\section{Introduction}

The decision whether or not to discharge an outpatient has major implications for the quality of patient care and efficiency of outpatient services [1-5]. This is particularly important in the primarily outpatient specialty of dermatology, where very large numbers of patients are seen, and delayed discharge of patients has a major impact on service efficiency. Both patients and the health service can be adversely affected by inappropriately early outpatient discharge and conversely by inappropriate follow-up appointments.
Prof. Andrew Y. Finlay

School of Medicine, Cardiff University

3rd Floor, Glamorgan House

Heath Park, Cardiff CF14 4XN (UK)

E-Mail FinlayAY@cf.ac.uk 
Table 1. Key methods for discharging outpatients, based on 3 qualitative studies [3-5]

1. Have discharge in mind in all consultations

2. At first appointment set expectations for discharge

Explain the likely course of treatment and expected outcomes (e.g. acne or laser patients may have predictable numbers of clinic appointments)

3. Discuss possible discharge "at the next visit" and document it in patient's notes

4. Ask patients directly if they want to come back to the clinic

Be firm and directive where appropriate

5. Explore the patient's agenda

Ask patients what they were hoping for from the consultation

Take more time when handling a "difficult" patient

6. Be honest if you have nothing more to offer the patient

7. Allow extra time to explain reasons and plan the discharge with the patient

Time is the key to a successful discharge

Clinic numbers may need to be reduced, but compensated by a higher rate of discharge

8. Inform and summarise events and investigation results in a positive manner

Remind patients of the positive outcomes from their clinic visits

Remind patients that we do not want them to have unnecessary clinic visits

9. Prepare patients to self-manage

Educate patients and provide information about realistic outcomes

Provide information to help patients self-manage, e.g. information leaflets or website links

Give positive feedback, e.g. praising the patient for learning about their disease and taking more

responsibility for management

10. Provide patients with a safety net

Make clear that care is not ending but will continue with transfer back to the GP

Encourage patients to seek help from their GP and explain the circumstances when the GP would

re-refer them back to the clinic

Use e-mail to communicate with patients after "simple" surgical procedures, e.g. uncomplicated basal cell carcinoma in low-risk site; note that e-mailing patients may have medicolegal implications [13]

Allow direct patient contact with the consultant's secretary, especially with anxious patients

If there is a dermatology department specialist nurse advice service, explain to patients when and how to access this service

Consider giving the patient an "open access" appointment

11. Provide GP with a clear management plan; consider copying this "discharge" letter to the patient

Check that the GP can prescribe your recommended therapy or carry out your suggested plan

Dictate the discharge letter in front of the patient

Most clinicians have never been taught how to discharge an outpatient, and most trainees receive no discharge training [2]. Despite outpatient discharge being one of the commonest decisions taken by clinicians there has been very little research aimed at understanding it. Training on how to discharge is essential to ensure that appropriate decisions are made in the best interests of the patient. Training is also necessary because of long waiting lists for outpatient specialist care $[6,7]$, uncertainties over how to make discharge decisions $[8,9]$, patients' lack of understanding of discharge instructions $[10,11]$, mismatched perception during discharge discussions $[4,10]$ and because of delays in re-accessing secondary care [4].

Discharge decision-making is a specific decision skill encompassing clinical expertise, communication skills, weighing of ethical issues, and ability to organise clinical practice. It needs to be taught to improve the quality of care and to reduce medical errors. Education research has focused on the most effective ways to teach clinicians how to make appropriate diagnostic and treatment decisions [12], but discharge decision-making has largely been ignored. What little has been published has focused on educating nurses, patients and family members [11] with little emphasis on educating clinicians. Vreeland et al. [11] suggested that inpatient discharge education should be structured, individualised, include the patient's preferences, include family involvement, provide multimedia instructions, emphasise medication intake, and include education of the multidisciplinary team involved in inpatient discharge: clinicians, nurses, dieti- 
Table 2. The 13 items of the "traffic light" design discharge information checklist [5]

\begin{tabular}{lll}
\hline Disease related & Patient empowerment & Addressing concerns \\
\hline Am I certain of the diagnosis? & $\begin{array}{l}\text { Has the patient understood the } \\
\text { diagnosis and treatment outcome? }\end{array}$ & Has the patient any concerns? \\
Is the condition severe? & $\begin{array}{l}\text { Have I explained to the patient a } \\
\text { clear plan of treatment? }\end{array}$ & $\begin{array}{l}\text { Can the patient re-access } \\
\text { secondary care easily if the } \\
\text { problem recurs? }\end{array}$ \\
\hline $\begin{array}{l}\text { Is the patient under the } \\
\text { appropriate treatment? }\end{array}$ & $\begin{array}{l}\text { Have I explained the treatment side } \\
\text { effects? }\end{array}$ & $\begin{array}{l}\text { Is the patient happy to be } \\
\text { discharged? }\end{array}$ \\
\hline $\begin{array}{l}\text { Can the patient be managed in } \\
\text { primary care? }\end{array}$ & $\begin{array}{l}\text { Has the patient understood how to } \\
\text { self-manage? }\end{array}$ & $\begin{array}{l}\text { Am I happy to discharge the } \\
\text { patient? }\end{array}$ \\
\hline
\end{tabular}

Will the patient benefit from my

follow-up?

cians, and social workers. They concluded that discharge education reduced re-admissions and lowered health care costs [11].

We have recently reviewed the literature [2] and reported dermatologists' views on influences on the outpatient discharge decision [3] and patients' views on being discharged [4]. We proposed a "traffic light" design dermatology outpatient discharge information checklist to ensure that the minimum information to take a quality discharge decision is available to the clinician [5]. During this qualitative research project, consultants and patients made many suggestions over the discharge process and about key aspects that could be taught to trainee doctors.

The aim of this paper is to provide recommendations, bringing together lessons from these prior studies that are of direct relevance to the education of clinicians concerning outpatient discharge decision-making. This paper does not present novel research findings, but encapsulates the educational and training issues arising from recent research [2-5].

\section{Background}

The educational recommendations given in this paper are based on information gathered in 3 qualitative studies of consultant and patient perspectives of dermatology outpatient discharge [3-5]. Ethical permission was given for the gathering of educational information during these studies. Forty dermatologists working in 11 hospitals were interviewed [3]: they were asked on how they arrived at a discharge or follow-up decision, what critical factors influenced their decision, what they considered an "appropriate" discharge and were asked to suggest strategies on how junior clinicians should be taught to take appropriate discharge decisions. Fifty-six patients with a range of dermatology conditions were interviewed after a routine dermatology outpatient consultation at which a decision was reached about whether or not they should be discharged [4]. In order to identify the most important pieces of information that a clinician should know to take an appropriate outpatient discharge decision [5], 17 dermatologists took part in a 3-stage Delphi process that led to the creation of a traffic light design checklist [5].

\section{Educational Recommendations}

\section{Methods to Improve the Process of Outpatient Discharge}

Key methods to improve the process of outpatient discharge, based on the 3 qualitative studies [3-5], are given in Table 1. Key aspects of an appropriate discharge decision are given in Figure 1.

\section{Methods to Inform the Discharge Decision to Ensure Its Appropriateness}

The 13 items (Table 2) of the traffic light design discharge checklist [5] can be used as a teaching tool. In the Delphi process that led to this checklist, some items did not qualify as part of the discharge checklist but are still useful to consider in specific clinical contexts during the discharge decision-making process (Table 3). During the studies, consultants suggested several methods on how dermatology trainees could be trained to take discharge decisions. Table 4 summarises these methods, illustrated with relevant consultant quotes. 
Table 3. Other items to be considered when making a discharge decision, in addition to those in the "traffic light" checklist

\begin{tabular}{ll}
\hline Items to be considered & Clinical context \\
\hline Patient's psychological state of mind & Patients who are anxious \\
\hline Complexity of the skin disease & Patients with comorbidities \\
\hline Chronicity of the skin disease & Patients whose diseases remit and recur \\
\hline Skin disease impact on the patient's quality of life & Patients with seemingly poor quality of life \\
\hline Nature of the patient's work & Patients with tight work schedules \\
\hline Mobility of the patient & Elderly patients or patients who live far from the hospital \\
\hline Presence of a carer & Mainly in children or vulnerable patients \\
\hline Patient-carer or patient-parent relationship & Mainly in children or vulnerable patients \\
\hline Quality of the patient-GP relationship & $\begin{array}{l}\text { Patients insisting on follow-up or unwilling to seek care from } \\
\text { their GP }\end{array}$ \\
\hline Local or national guidelines & When faced with clinical uncertainty \\
\hline $\begin{array}{l}\text { Appropriate management support from other care providers } \\
\text { such as oncologists, psychologists }\end{array}$ & Patients with comorbidities \\
\hline Health care service financial resources & Limited health care budget \\
\hline $\begin{array}{l}\text { Local discharge policy } \\
\text { Referral of the patient to nurse-led clinics in secondary care }\end{array}$ & Relevant clinic discharge policy \\
\hline
\end{tabular}

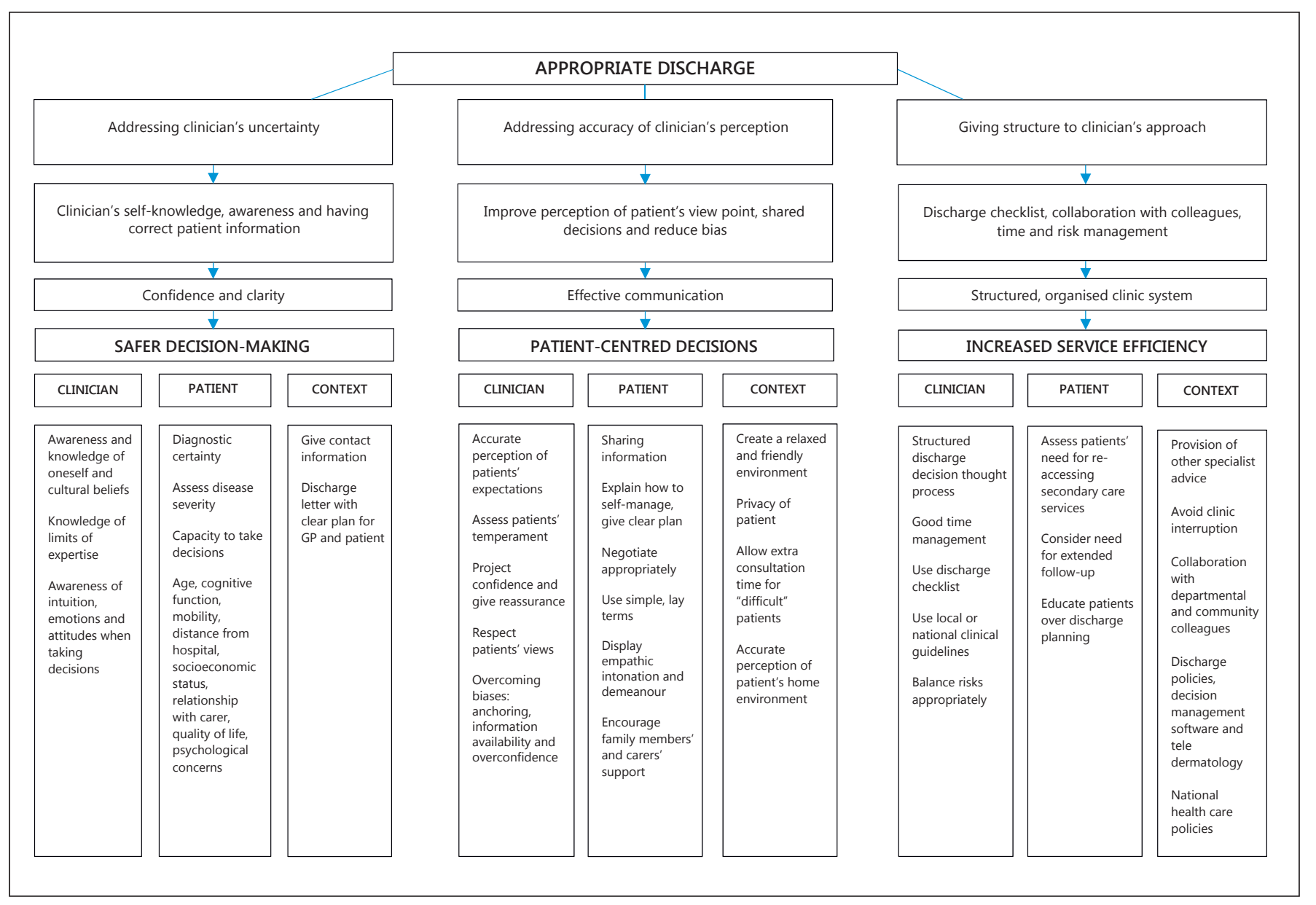

Fig. 1. Key aspects of an appropriate discharge. 
Table 4. Methods to train dermatology trainees how to discharge outpatients

\begin{tabular}{|c|c|}
\hline Training tips & Consultant quotations \\
\hline Learning through modelling a senior consultant & $\begin{array}{l}\text { "Learning to discharge is by example rather than } \\
\text { some written guideline" }\end{array}$ \\
\hline Supervised "hands-on" experience & $\begin{array}{l}\text { "You have to teach them how to discharge patients: as } \\
\text { I have said before, whether they are safe to be } \\
\text { discharged, are they stabilized, and is the GP able to } \\
\text { monitor them, I think you've got to make a decision } \\
\text { to discharge patients!" }\end{array}$ \\
\hline $\begin{array}{l}\text { Review by consultant of every patient who is } \\
\text { discharged by a trainee }\end{array}$ & $\begin{array}{l}\text { "I checked the [trainee's] letters back to GPs, } \\
\text { explaining how to give them a definite treatment plan, } \\
\text { and suggesting what to do in this situation" }\end{array}$ \\
\hline Learn to access and use relevant "guidelines" & $\begin{array}{l}\text { "I guess if we were perhaps more fully supported with } \\
\text { specific guidelines for specific conditions, in terms of } \\
\text { exact follow-up guidance, we felt supported by that" }\end{array}$ \\
\hline $\begin{array}{l}\text { Encourage self-confidence and acting on the basis } \\
\text { of clinical judgement }\end{array}$ & $\begin{array}{l}\text { "If I really thought clinically that the patients did not } \\
\text { really need to be coming back for a clinic } \\
\text { appointment, I would make a real effort to discharge } \\
\text { them. I would try to explain to them clearly why they } \\
\text { didn't need to come" }\end{array}$ \\
\hline Collaborate and communicate well as a team & $\begin{array}{l}\text { "I guess this reflects on us as the supervising clinic, so } \\
\text { good communication between juniors and seniors } \\
\text { about which patients need to be followed up" }\end{array}$ \\
\hline
\end{tabular}

Table 5. Proposed educational objectives concerning the outpatient discharge decision

To adopt a "shared decision-making" approach rather than a paternalistic approach

To self-recognise and manage poor verbal and non-verbal communication with patients

To recognise and manage ineffective collaboration with colleagues in primary and secondary care

To demonstrate confidence when making discharge decisions

To use the "traffic light" design discharge information checklist during training and routinely where appropriate To evaluate strategies for appropriate discharge decisions

To recognise biases related to taking discharge decisions, such as overconfidence and anchoring biases

To manage consultation times to ensure sufficient time available for the discharge process

To reflect on the appropriateness of clinical decisions taken either to discharge or follow up outpatients

\section{Proposed Educational Objectives}

Since outpatient discharge is the critical end point of patient care in the outpatient clinical setting, appropriate discharge decisions are not only important for the well-being of individual patients, but are integral to ensuring health care service efficiency. The purpose of educating clinicians about outpatient discharge is to enable them to make high-quality dermatology outpatient discharge decisions, with the aim of ensuring that no patient is disadvantaged as a result of a clinician's lack of confidence and competence in taking discharge decisions. We propose a series of broad educational objectives (Table 5).

\section{Discussion}

We have developed novel strategies on how to appropriately manage the outpatient discharge process, inform and make discharge decisions. There are 3 main aspects: safer decision-making (self-awareness, knowledge on limits of own expertise, patients' characteristics, diagnostic certainty, providing contact after discharge, clear treatment plan), patient-centred decisions (accurate perception and management of patients' expectations, reducing clinician biases, longer consultation time for "difficult" patients, effective communication), and increased service efficiency (tool to guide discharge decisions, use 
of clinical guidelines, good time management, effective collaboration with colleagues and general practitioners, provision of open appointments and discharge policies). These strategies are similar to those identified in a qualitative study of discharge from general medical outpatients [10].

Factors of most importance to patients in being satisfied with their dermatology outpatient consultation have been identified as physician's concern about and focus on quality of life, physician's interest in patients' symptoms and medical problems, and the carrying out of full skin examination or specialised examination such as use of a dermatoscope [14]. That study however did not investigate satisfaction with the discharge process. In a study of dermatology outpatients immediately after being discharged, we identified considerable dissatisfaction amongst some patients at being discharged, even though they had apparently agreed to the discharge during the consultation [4].

There is a distinction between what actions are critical to the process of making an appropriate discharge and how best to inform patients about the discharge decision itself. Both these aspects of patient discharge involve a set of decision skills that could be taught in a structured manner [2-5].

The consideration of a wide array of clinical and nonclinical factors influencing the discharge process can make judgement difficult for the inexperienced clinician who may inadvertently be subject to inappropriate biases, often without insight $[2,3]$. Junior doctors should be educated on the likely existence of bias, in order to support better clinical decision-making [12, 15].

In many health care systems there is great pressure on clinical time, with restricted consultation length. It may also take longer to discharge a patient than to offer a follow-up appointment. However, unnecessary followup appointments may be a major contributing factor to the time pressures on dermatology outpatient clinics as every unnecessary appointment is a lost opportunity to see a new patient or to spend longer time with other patients. Training in outpatient discharge decision-making may therefore contribute appropriately to increased service efficiency. Most clinicians have never been trained to take outpatient discharge decisions but have learnt themselves. Some clinicians may have little insight into the influences on their decision-making and little awareness of the biases that they bring to clinical decision-making. This may contribute to scepticism of the possible benefits of training in discharge decisionmaking.

How to Discharge an Outpatient
One limitation of the information on which the educational recommendations are made is that the information came primarily from detailed interviews with consultant dermatologists and with patients, but without input from general practitioners, other health care professionals or from dermatology trainees. Further research gaining the perspective of these other groups [16] would be of benefit. The research on which these educational recommendations were based was carried out in the context of the UK publically funded National Health Service. While many of the influences on dermatology outpatient discharge decisions may be encountered in any health care system, it is possible that there may be other influences and different strategies to be considered in, for example, a private dermatology clinic. We are not aware of any research on this topic carried out in a private health care setting.

There are many different patterns of private clinical practice both within and between different countries, with widely differing rates of outpatient discharge. In private practice, the dynamics between patient and physician may differ from public practice, with the additional influence of consultation fees. However, even in private practice it may be helpful for the physician to have received training in clinical strategies relating to taking appropriate discharge decisions and in managing the process of discharge to the satisfaction of the patient. It remains to be tested whether the concepts and strategies we have described may be appropriate for use in private practice.

One educational objective is to encourage clinicians to consider the items in the traffic light design discharge information checklist [5] and to use the checklist routinely in outpatient practice. The checklist covers 5 disease-related items, 4 patient empowerment items and 4 patient concerns, which if considered, may encourage earlier appropriate discharge or prevent inappropriately early discharge. The 5 disease-related items are in the red (stop) section of the checklist. These indicate a command to the clinician to stop and seriously consider these items before proceeding. When these have been considered, the clinician proceeds to the next step, considering the 4 patient empowerment items in the yellow (proceed with caution) section. After this, the clinician should consider addressing concern items in the green (permission to proceed) section. When all of these items have been addressed, the patient can be discharged [5]. Although it was designed to be concise and user friendly, the use of the checklist as a practical tool in the clinic or in education has yet to be tested. The use of the checklist may be the method most

Dermatology 2017;233:260-267 DOI: $10.1159 / 000479060$ 
likely to ascertain discharge appropriateness, and its use may help to minimise biases due to personal judgement and cognitive errors, which recur predictably in discharging patients. We suggest prospective research to ascertain whether the use of this discharge information checklist and education in this area can make any difference to the quality of outpatient discharge decision-making. It is possible that both the discharge information checklist and suggested educational goals and strategies may be used as a platform to improve discharge practice in other specialties or also in inpatient discharge practice.

When first encountered, the traffic light checklist approach may seem unfeasible. However, in a small survey in a publicly funded dermatology clinic [5], 61\% of 18 clinicians reported that they would like to use the traffic light checklist. The traffic light concept has not been used in private practice. Prospective research is required to test whether an adaptation of the concept or perhaps a different approach may be needed for the private practice setting.

There is not yet sufficient evidence to support the traffic light system being a requirement for training. However, training concerning discharge decision-making should be a requirement, as this is one of the commonest clinical decisions that every dermatologist takes on a daily basis. Whether or not it is used in clinical practice, the traffic light checklist may be useful to focus discussion and training in this area.

A radically different alternative approach to reducing outpatient clinic numbers by increasing discharge decision rates has been suggested [17]. A senior clinician reviewed all outpatient charts 2 weeks before the clinic, and a brief written management plan was made. This resulted in a large reduction in clinic sizes. It would be possible to use such a review as a teaching exercise for trainees.

It is important to encourage trainees to reflect [18] on how well they handle judgement under uncertainty, how effectively they communicate and to have insight into their unconscious use of mental shortcuts that may cause biased decisions. Over a century ago, the Flexner report [19] stressed that medical students should be taught how to apply clinical reasoning when making decisions when faced with uncertainty, and the discharge decision is a good example on which to base education about this issue. Clinicians are often immersed in the technicalities of disease management and therefore may neglect the more "humane" aspects of patient care. They may fail to realise that during consultations patients are affected by the attitudes of their clinician $[4,10,20]$. Teaching trainee clinicians to actively reflect over each of their patient con- sultations may assist trainees to project an effortless empathic response to patients. Such skills are learned partly from experience and need frequent self-reflection to maintain and improve them. Because of the often incurable nature and high visibility of skin diseases, trainee dermatologists need to be more aware of the needs of patients with skin disease and whether a patient is actually ready for discharge.

Other aspects of routine clinical decision-making such as diagnostic and treatment decisions should also be taught, rather than assuming that clinicians will learn by imperceptibly "soaking up" the experience of seniors in an apprentice relationship. These wider decisions should be examined in a similar way, to help transform the "art of medicine" into an approach less liable to bias. It is appropriate to try to improve the quality of a clinician's approach to taking clinical decisions, since clinicians exert great influence on their patients and the health care team. It is also important for those who make decisions about groups of patients or populations to learn a more structured and transparent approach to making evidencebased decisions. Integrating training in decision-making into dermatology trainee programmes will hopefully improve the quality of patient care.

\section{Conclusion}

This paper summarises a novel educational approach to the process of making outpatient discharge decisions, based on qualitative research evidence. The time has come to revisit and sharpen our skills over discharging outpatients in an attempt to minimise biases and uncertainties. When genuine effort, professional wisdom and patient empowerment come together, the goal of making more appropriate discharge decisions may then be realised. If dermatologists better understood how discharge decisions should be carried out, greater discharge decision appropriateness might be achieved in the best interests of patients and dermatology services. Furthermore, it is hoped that educators in medicine will seriously consider inclusion of "outpatient discharge decision-making" in the mainstream curriculum.

\section{Key Message}

This review describes how to educate trainees to make appropriate high-quality outpatient discharge decisions.
Harun/Finlay/Salek/Piguet 


\section{Acknowledgements}

We wish to thank the dermatologists and dermatology patients whose ideas are summarised in this article. We also wish to thank clinicians from the University Hospital of Wales (UHW), Cardiff, who contributed suggestions on making outpatient discharge decisions at meetings organised by A.Y.F. in 2008-2009, incorporated in Table 1.

\section{Disclosure Statement}

All authors state no conflict of interest.

\section{References}

1 Sullivan FM: How do clinicians decide to discharge someone from their out-patient clinic? J Manag Med 1993;7:24-28.

2 Harun NA, Salek S, Piguet V, Finlay AY: The dermatology outpatient discharge decision: understanding a critical but neglected process. Br J Dermatol 2014;170:1029-1038.

3 Harun NA, Finlay AY, Salek MS, Piguet V: Appropriate and inappropriate influences on outpatient discharge decision making in dermatology: a prospective qualitative study. $\mathrm{Br} \mathrm{J}$ Dermatol 2015;173:720-730.

4 Harun NA, Finlay AY, Piguet V, Salek S: Understanding clinician influences and patient perspectives on outpatient discharge decisions: a qualitative study. BMJ Open 2017; 7:e010807.

5 Harun N, Finlay A, Salek MS, Piguet V: The development and clinical evaluation of a "traffic-light design" dermatology outpatient discharge information checklist. Br J Dermatol 2016;175:572-582.

6 Salek MS, Siyani S, Basra MK, Finlay AY: The clinical and non-clinical factors influencing discharge decisions in dermatology: is there a need for discharge strategy? Int J Clin Pharmacol 2012;34:178-179.
7 Stainkey LA, Seidl IA, Johnson AJ, et al: The challenge of long waiting lists: how we implemented a GP referral system for non-urgent specialist appointments at an Australian public hospital. BMC Health Serv Res 2010;10:1.

8 Burkey Y, Black M, Reeve H, et al: Long-term follow-up in outpatient clinics. 2. The view from the specialist clinic. Fam Pract 1997;14: 29-33.

$9 \mathrm{Wu} \mathrm{K}-\mathrm{H}$, Chen IC, Li C-J, et al: The influence of physician seniority on disparities of admit/ discharge decision making for ED patients. Am J Emerg Med 2012;30:1555-1560.

10 Burkey Y, Black M, Reeve H: Patients' views on their discharge from follow up in outpatient clinics: qualitative study. BMJ 1997;315: 1138-1141.

11 Vreeland D, Rea RE, Montgomery L: A review of the literature on heart failure and discharge education. Crit Care Nurs Q 2011;34:235245.

12 Hunink MM, Weinstein MC, Wittenberg E, et al: Decision Making in Health and Medicine: Integrating Evidence and Values. Cambridge, Cambridge University Press, 2014

13 Brahams D: The medicolegal implications of teleconsulting in the UK. J Telemed Telecare 1995;1:196-201.
14 De Salins CA, Brenaut E, Misery L, RoguedasContios AM: Factors influencing patient satisfaction: assessment in outpatients in dermatology department. J Eur Acad Dermatol Venereol 2016;30:1823-1828.

15 Croskerry P, Norman G: Overconfidence in clinical decision making. Am J Med 2008; 121:S24-S29.

16 Reeve H, Baxter K, Newton P, Burkey Y, Black $\mathrm{M}$, Roland M: Long term follow-up in outpatient clinics. 1. The view from general practice. Fam Pract 1997;14:24-28.

17 Donnellan F, Hussain T, Aftab AR, McGurk C: Reducing unnecessary outpatient attendances. Int J Health Care Qual Assur 2010;23: 527-531.

18 Magnussen L, Trotter CM: The reflective clinician. Nurse Educ 1997;22:40-44

19 Flexner A: 1910. Medical education in the United States and Canada. http://archive. carnegiefoundation.org/pdfs/elibrary/ Carnegie_Flexner_Report.pdf (accessed Apri 16, 2017).

20 Agledahl KM, Gulbrandsen P, Førde R, et al: Courteous but not curious: how doctors' politeness masks their existential neglect. A qualitative study of video-recorded patient consultations. J Med Ethics 2011;37:650-654. 